\title{
Inverse Compton radiation from GRB afterglows in the VHE range
}

\author{
Jagdish C. Joshi* \\ Centre for Astro-Particle Physics (CAPP) and Department of Physics, University of \\ Johannesburg, PO Box 524, Auckland Park 2006, South Africa \\ E-mail: jjagdish@uj.ac.za

\section{Soebur Razzaque} \\ Centre for Astro-Particle Physics (CAPP) and Department of Physics, University of \\ Johannesburg, PO Box 524, Auckland Park 2006, South Africa \\ E-mail: srazzaque@uj.ac.za
}

\begin{abstract}
The observation of Gamma-ray bursts (GRBs) can be classified into prompt and afterglow emission. The prompt emission is observed for up to 100 s of seconds after the trigger of the event while a delayed and long-lasting afterglow emission is followed after this phase. The prompt emission dominates in the $\gamma$-rays ( $\mathrm{keV}-\mathrm{MeV}-\mathrm{GeV}$ ), while the afterglow emission mainly occurs in X-ray, $\mathrm{UV}$, optical, radio wavelengths and possibly in $\gamma$-rays. In this work, we study the signature of inverse Compton emission in the afterglow emission of GRBs. We consider the expansion of an adiabatic blast wave into a constant density medium and use the observation of GRB 130427A afterglow to constrain the model parameters. The flux produced via inverse-Compton mechanism have been discussed and also possible detection by the Cherenkov Telescope Array mainly in the range $30 \mathrm{GeV}-1 \mathrm{TeV}$ have been discussed.
\end{abstract}

6th Annual Conference on High Energy Astrophysics in Southern Africa

1-3 August, 2018

Parys, Free State, South Africa

${ }^{*}$ Speaker. 


\section{Introduction}

Gamma ray bursts (GRBs) are energetic explosions, which indicates either death of a massive star $\left(>25 M_{\odot}\right)$ or merger of two compact objects in combination of a neutron star and a black-hole [1 -4]. In our current understanding, the GRB population follows a bimodal distribution based on which, collapsar-based GRBs stay active for long duration $\left(T_{90}>2 \mathrm{~s}\right)$, and merger-based GRBs last for very short duration $\left(T_{90}<2 \mathrm{~s}\right)[5,6] . T_{90}$ is the time over which a GRB emits $90 \%$ of its total measured fluence.

The Fermi gamma-ray space telescope have a keV-30 MeV gamma-ray burst monitor (GBM) [7] and $20 \mathrm{MeV}-300 \mathrm{GeV}$ large area telescope (LAT) detector [8]. LAT emission from GRBs is delayed compared to the low-energy GBM detection [9], and this emission extends to tens of $\mathrm{GeV}$ energies, for example in GRB 130427A, a photon of energy 95-GeV was detected after $244 \mathrm{~s}$ of the trigger [10]. For GRB 130427A, in our earlier work [11], we have discussed the origin of the extended component could be the Fe-nuclei disintegration but the same mechanism can have very high luminosity requirement for GRB 090926A and GRB 090902B [12]. The synchrotron emission from GRBs, when the blast wave expands into the interstellar medium can explain the multi-wavelength afterglow observations [13], but remains challenging to produce very high energy (VHE), above $10 \mathrm{GeV}$ emission.

In this work, we have studied the expansion of the GRB blast wave into the interstellar medium with constant density and apply flux models to GRB 130427A data. We have also studied the prospects for inverse Compton (IC) emission detection by ground based Cherenkov Telescope Array (CTA). CTA will have two set of imaging atmospheric Cherenkov telescope (IACT), one in the northern hemisphere (La Palma, Spain) and the other in the Southern hemisphere in Chile. Based on the Fermi-LAT observation of GRBs the optimistic detection rate at CTA can be 1-2 events per year [14]. In our model, the parameters are the density of the ambient medium $n_{0}$, blast wave kinetic energy $E_{k}$, microphysical parameters $\varepsilon_{e}, \varepsilon_{B}$, which represent the fraction of jet kinetic energy in the electrons and magnetic field respectively. Sensitivity of the CTA can access the GeV-TeV range of energy from GRBs and can provide us more detail about the jet and microphysical parameters.

\section{GRB blast-wave model and the afterglow radiation}

The blast-wave from GRB events expands into the circum-burst medium (CBM) and decelerates significantly, when the swept-up mass is comparable in energy with the injected kinetic energy $E_{k}$ in the blast-wave. The swept-up mass at deceleration radius $r_{d}$ from spherically symmetric blast-wave is $M_{\mathrm{sw}}=4 \pi r_{d}^{3} m_{p} n_{0} / 3$, where $n_{0}$ is the ambient medium density. The energy in the swept-up mass in the stationary frame would be $\Gamma_{0}^{2} M_{\mathrm{sw}} c^{2}$, where $\Gamma_{0}$ is the initial Lorentz factor of the blast-wave. From the condition $E_{k}=\Gamma_{0}^{2} M_{\mathrm{sw}} c^{2}$, the deceleration radius is [15],

$$
r_{d}=\left(\frac{3 E_{k}}{4 \pi \Gamma_{0}^{2} m_{p} c^{2} n_{0}}\right)^{1 / 3}=2.51 \times 10^{17} n_{0}^{-1 / 3} \Gamma_{2.5}^{-2 / 3} E_{55}^{1 / 3} \mathrm{~cm}
$$

Where $E_{k}=10^{55} E_{55}$ erg is the isotropic-equivalent kinetic energy of the blast-wave and $\Gamma_{0}=$ $10^{2.5} \Gamma_{2.5}$ is Lorentz factor at the trigger. The deceleration time is defined as $t_{d}=(1+z) r_{d} / 2 \Gamma_{0}^{2} c$, 


$$
t_{d}=\left(\frac{3 E_{k}(1+z)^{3}}{32 \pi n_{0} m_{p} c^{5} \Gamma_{0}^{8}}\right)^{1 / 3}=42.2(1+z) n_{0}^{-1 / 3} \Gamma_{2.5}^{-8 / 3} E_{55}^{1 / 3} \mathrm{~s},
$$

The Lorentz factor of the blast-wave evolves with time as $\Gamma(t)=\Gamma_{0}\left(t_{\mathrm{dec}} / 4 t\right)^{3 / 8}$, and the radius after deceleration time $t=t_{\mathrm{d}}$, can be estimated using the time evolution of the bast wave radius [16] as

$$
\begin{aligned}
\Gamma(t) & =136.1(1+z)^{3 / 8} n_{0}^{-1 / 8} E_{55}^{1 / 8} t_{2}^{-3 / 8} \\
R(t) & =\frac{2 \Gamma^{2}(t) a c t}{1+z}=4.44 \times 10^{17}(1+z)^{-1 / 4} n_{0}^{-1 / 4} E_{55}^{1 / 4} t_{2}^{1 / 4} \mathrm{~cm}
\end{aligned}
$$

where $a=4$ for an adiabatic blast-wave and $t_{2}=t / 100 \mathrm{~s}$. In the blast-wave collision with the ISM, external shocks are produced which accelerate the electrons. The electron distribution assumed to follow a power law distribution, with spectral-index p, $N_{e} d \gamma_{e} \propto \gamma_{e}^{-p} d \gamma_{e}$. The electron Lorentz factor $\gamma_{m}^{\prime}, \gamma_{c}^{\prime}$ and $\gamma_{s}^{\prime}$ in the jet frame are discussed below. The synchrotron cooling time is $t_{\mathrm{sy}}^{\prime}=$ $3 m_{e} c^{2} /\left(4 \sigma_{T} c \gamma_{e}^{2} U_{B^{\prime}}\right)$, and inverse-Compton cooling time is $t_{\mathrm{IC}}^{\prime}=3 m_{e} c^{2} /\left(4 \sigma_{T} c \gamma_{e}^{2} U_{\mathrm{ph}^{\prime}}\right)$, where $U_{B^{\prime}}$ is the magnetic energy density in the jet and $U_{\mathrm{ph}^{\prime}}$ is the target photon energy density produced by synchrotron emission. The cooling Lorentz factor can be estimated by comparing the total cooling time $t_{c}^{\prime}=\left[1 / t_{\mathrm{sy}}^{\prime}+1 / t_{\mathrm{IC}}^{\prime}\right]^{-1}$ with the dynamic or expansion time scale $t_{d y n}^{\prime}=\Gamma /(1+z) t$ as

$$
\gamma_{c}^{\prime}(t)=\left[\frac{6 \pi m_{e} c^{2}(1+z)}{\sigma_{T} c B^{\prime 2}(t) t \Gamma(t)(1+Y)}\right]
$$

In this work the Compton parameter has been modelled as $Y=\left(\varepsilon_{e} / \varepsilon_{B}\right)^{1 / 2}$, which is valid for fast cooling [17]. The saturation Lorentz factor comes by comparing the accelerating time scale $t_{a c c}^{\prime}=\phi \gamma_{e}^{\prime} m_{e} c / e B^{\prime}(t)$, where acceleration efficiency $\phi=10 \phi_{1}$, with the total cooling time $t_{c}^{\prime}$ and is given by

$$
\gamma_{s}^{\prime}(t)=\left[\frac{6 \pi e}{\phi \sigma_{T} B^{\prime}(t)(1+Y)}\right]
$$

The minimum Lorentz factor of the electrons for $p>2$ [13] is

$$
\gamma_{m}^{\prime}(t)=\left[\frac{m_{p}}{m_{e}} \varepsilon_{e} \frac{p-2}{p-1} \Gamma(t)\right]
$$

Electrons with these Lorentz factors cool at the external shock and radiate photons, the frequency of these radiated photons are listed below [17, 18]. The synchrotron-self absorption frequency is discussed in detail in [18], while the other three break fequencies are estimated using the cooling frequency [16] as

$$
h v(t)=\frac{3}{2} \frac{B^{\prime}(t)}{B_{Q}} \gamma^{\prime 2} m_{e} c^{2} \frac{\Gamma(t)}{1+z}
$$


where $B^{\prime}(t)=\left[32 \pi \varepsilon_{B} n_{0} m_{p} c^{2}\right]^{1 / 2} \Gamma(t)$ and $B_{Q}=4.41 \times 10^{13} \mathrm{G}$. The synchrotron break frequencies are, with $\varepsilon_{e}=0.1 \varepsilon_{e,-1}$ and $\varepsilon_{B}=0.1 \varepsilon_{B,-1}$.

$$
\begin{aligned}
v_{a}^{\mathrm{f}} & =4.3 \times 10^{12}\left(\frac{(p+2)(p-1)}{(3 p+2)}\right)^{3 / 5}(1+z)^{-1 / 2} \varepsilon_{B,-1}^{6 / 5} n_{0}^{11 / 10} E_{55}^{7 / 10} t_{2}^{-1 / 2}(1+Y) \mathrm{Hz}, \\
v_{c} & =5.64 \times 10^{14}(1+z)^{-1 / 2} \varepsilon_{B,-1}^{-3 / 2} n_{0}^{-1} E_{55}^{-1 / 2} t_{2}^{-1 / 2}(1+Y)^{-2} \mathrm{~Hz} \\
v_{m} & =4.18 \times 10^{18}\left(\frac{p-2}{p-1}\right)^{2}(1+z)^{1 / 2} \varepsilon_{B,-1}^{1 / 2} \varepsilon_{e,-1}^{2} E_{55}^{1 / 2} t_{2}^{-3 / 2} \mathrm{~Hz} \\
v_{s} & =7.12 \times 10^{23}(1+z)^{-5 / 8} \phi_{1}^{-1} n_{0}^{-1 / 8} E_{55}^{1 / 8} t_{2}^{-3 / 8}(1+Y)^{-1} \mathrm{~Hz}
\end{aligned}
$$

Using these set of frequencies we have calculated the synchrotron flux in the fast cooling phase with order of frequency $v_{a}<v_{c}<v_{m}<v_{s}$ which are describes as [13]

$$
F_{v}^{f}=f_{\max }\left\{\begin{array}{lc}
\left(v / v_{a}\right)^{2}\left(v_{a} / v_{c}\right)^{1 / 3} ; & v<v_{a} \\
\left(v / v_{c}\right)^{1 / 3} ; & v_{a}<v<v_{c} \\
\left(v / v_{c}\right)^{-1 / 2} ; & v_{c}<v<v_{m} \\
\left(v_{m} / v_{c}\right)^{-1 / 2}\left(v / v_{m}\right)^{-p / 2} \exp \left(-v / v_{\mathrm{s}}\right) ; \quad v \geq v_{\mathrm{m}}
\end{array}\right.
$$

the maximum synchrotron flux is, $f_{\max }=2.6(1+z) \varepsilon_{B,-1}^{1 / 2} E_{55} n_{0}^{1 / 2} D_{L, 28}^{-2} \mathrm{Jy}$, where $D=10^{28} D_{L, 28} \mathrm{~cm}$ is the distance to the source. For $N$ number of electrons in the jet, the inverse Compton flux is taken as $f_{\max }^{\mathrm{IC}}=\sigma_{T} N / 4 \pi R^{2}=2 \times 10^{-8} n_{0}\left(R(t) / 10^{18}\right) f_{\max }$ and the inverse Compton break frequencies are $v_{a}^{\mathrm{IC}}=2 \gamma_{m}^{2} v_{a}, v_{m}^{\mathrm{IC}}=2 \gamma_{m}^{2} v_{m}$ and $v_{c}^{\mathrm{IC}}=2 \gamma^{2} v_{c}$ respectively, also described in [17].

\section{Calculation and Results}

We have considered the MeV-GeV component observed for GRB 130427A during 130.05$196.61 \mathrm{~s}$ in our modelling, also shown in Figure 1. We consider the kinetic energy of the blast-wave is $E_{k}=10^{55} \mathrm{erg}$, which is expanding into the ISM with density 8 atoms $/ \mathrm{cm}^{3}$. The red-shift $z=$ 0.34 , the electron spectral index $p=2.3$ and microphysical parameters $\varepsilon_{e}=0.4$ and $\varepsilon_{B}=0.0003$ respectively. The synchrotron and SSC fluxes for these parameters, which fit the observational data for GRB 130427A, is shown in Figure 1. We found that the slow cooling spectrum requires very high luminosity to explain these fluxes in GRB 130427A.

The VHE photons will attenuate in the internal photon radiation field as well in the extrabackground light (EBL). At different times 130,200,500,1000 s, as per our target photon distribution in Figure 1, the Lorentz factors are 106, 90, 64 and 49 while the blast-wave radius evolve in the range $2-4 \times 10^{17} \mathrm{~cm}$ with time. The internal attenuation has been calculated based on the formalism discussed in $[19,11,15]$ and for photons of energy $500 \mathrm{GeV}$ the opacity is approximately $10^{-3}$ and negligible. The attenuation in the extragalactic background light (EBL) for GRB $130427 \mathrm{~A}$ at $z=0.34$ allows the maximum energy of the observed photon approximately $300 \mathrm{GeV}$ [20], and this has been included as a cut-off in the inverse-Compton spectrum.

The inverse-Compton flux levels are compatible with the CTA sensitivity which is approximately $10^{-10} \mathrm{erg} / \mathrm{cm}^{2} / \mathrm{s}$, for an observation time window of 100's of seconds [21]. 


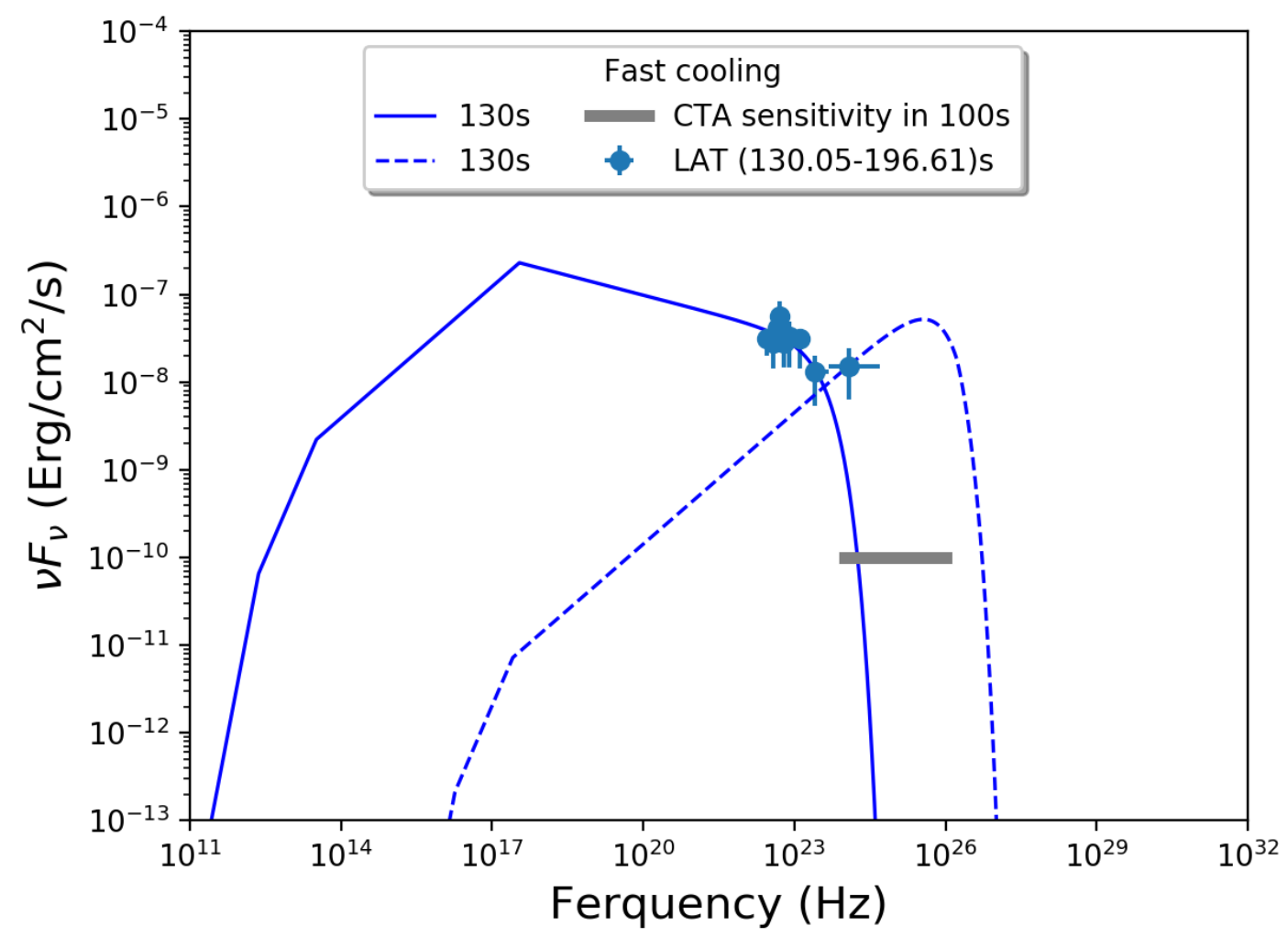

Figure 1: The synchrotron and inverse-Compton fluxes for GRB 130427A at different time intervals. The data points are taken from [22]. The synchrotron photon energy is limited by the saturation frequency $v_{s}$, while inverse-Compton component attenuation is calculated in the extra-background light (EBL) [20] as the internal attenuation is negligible. The CTA sensitivity is shown for the VHE detection [21].

\section{Conclusion and future Plan}

In our work, we have calculated the synchrotron and SSC emission for the scenario of GRB 130427A for an adiabatic blast-wave scenario at the external-shock. We showed that the early afterglow photons are produced by the synchrotron component and the upscattered inverse-Compton photons can produce the second peak in the spectrum. The sensitivity of CTA would discover these components in future if any GRB of type 130427A occurs during its operation.

\section{Acknowledgement}

This work was supported by grants from the National Research Foundation (NRF) South Africa.

\section{References}

[1] S. E. Woosley and J. S. Bloom, The Supernova Gamma-Ray Burst Connection, ARAA 44 (Sept., 2006) 507-556, [astro-ph/ 0609142 ]. 
[2] P. Ferrero, D. A. Kann, A. Zeh, S. Klose, E. Pian, E. Palazzi et al., The GRB 060218/SN 2006aj event in the context of other gamma-ray burst supernovae, A\&A 457 (Oct., 2006) 857-864, [astro-ph/0605058].

[3] N. Gehrels and S. Razzaque, Gamma-ray bursts in the swift-Fermi era, Frontiers of Physics 8 (Dec., 2013) 661-678, [1301.0840].

[4] E. Berger, Short-Duration Gamma-Ray Bursts, ARAA 52 (Aug., 2014) 43-105, [1311. 2603 ].

[5] C. Kouveliotou, C. A. Meegan, G. J. Fishman, N. P. Bhat, M. S. Briggs, T. M. Koshut et al., Identification of two classes of gamma-ray bursts, ApJL 413 (Aug., 1993) L101-L104.

[6] D. Lazzati and R. Perna, Short Gamma Ray Bursts: Marking the Birth of Black Holes from Coalescing Compact Binaries, in Physics of Relativistic Objects in Compact Binaries: From Birth to Coalescence (M. Colpi, P. Casella, V. Gorini, U. Moschella and A. Possenti, eds.), vol. 359 of Astrophysics and Space Science Library, p. 245, 2009. 1001.0538.

[7] C. Meegan, G. Lichti, P. N. Bhat, E. Bissaldi, M. S. Briggs, V. Connaughton et al., The Fermi Gamma-ray Burst Monitor, ApJ 702 (Sept., 2009) 791-804, [0 908.0450 ].

[8] W. B. Atwood, A. A. Abdo, M. Ackermann, W. Althouse, B. Anderson, M. Axelsson et al., The Large Area Telescope on the Fermi Gamma-Ray Space Telescope Mission, ApJ 697 (June, 2009) 1071-1102, [0902.1089].

[9] A. A. Abdo, M. Ackermann, M. Arimoto, K. Asano, W. B. Atwood, M. Axelsson et al., Fermi Observations of High-Energy Gamma-Ray Emission from GRB 080916C, Science 323 (Mar., 2009) 1688.

[10] M. Ackermann, M. Ajello, K. Asano, W. B. Atwood, M. Axelsson, L. Baldini et al., Fermi-LAT Observations of the Gamma-Ray Burst GRB 130427A, Science 343 (2014) 42-47.

[11] J. C. Joshi, S. Razzaque and R. Moharana, Photodisintegrated gamma rays and neutrinos from heavy nuclei in the gamma-ray burst jet of GRB 130427A, MNRAS 458 (2016) L79-L83.

[12] J. Joshi and S. Razzaque, Modeling GeV to Very High Energy Emission from Long GRBs, in PoS (HEASA 2016) 003, 25-26 August, 2016. South African Astronomical Observatory (SAAO), Cape Town, South Africa, p. 3, 2016.

[13] R. Sari, T. Piran and R. Narayan, Spectra and Light Curves of Gamma-Ray Burst Afterglows, ApJL 497 (Apr., 1998) L17-L20, [astro-ph/9712005].

[14] R. C. Gilmore, A. Bouvier, V. Connaughton, A. Goldstein, N. Otte, J. R. Primack et al., IACT observations of gamma-ray bursts: prospects for the Cherenkov Telescope Array, Experimental Astronomy 35 (Apr., 2013) 413-457, [1201 . 0010 ].

[15] C. D. Dermer and G. Menon, High Energy Radiation from Black Holes: Gamma Rays, Cosmic Rays, and Neutrinos, Princeton Univerisity Press. 2009.

[16] S. Razzaque, Long-lived PeV-EeV neutrinos from gamma-ray burst blastwave, PRD 88 (2013) 103003.

[17] R. Sari and A. A. Esin, On the Synchrotron Self-Compton Emission from Relativistic Shocks and Its Implications for Gamma-Ray Burst Afterglows, ApJ 548 (Feb., 2001) 787-799, [astro-ph/0005253].

[18] J. Granot, T. Piran and R. Sari, Synchrotron Self-Absorption in Gamma-Ray Burst Afterglow, ApJ 527 (Dec., 1999) 236-246, [astro-ph/9808007]. 
[19] R. J. Gould and G. P. Schréder, Opacity of the Universe to High-Energy Photons, Physical Review 155 (Mar., 1967) 1408-1411.

[20] J. D. Finke, S. Razzaque and C. D. Dermer, Modeling the Extragalactic Background Light from Stars and Dust, ApJ 712 (Mar., 2010) 238-249, [0 905.1115$].$

[21] CTA, "Sensitivity vs Observation Time, year $=2017$, url = https://www.cta-observatory.org/science/cta-performance/1525680063092-06388df6-d2af, urldate = 2019-21-01."

[22] W. T. Vestrand, J. A. Wren, A. Panaitescu, P. R. Wozniak, H. Davis, D. M. Palmer et al., The Bright Optical Flash and Afterglow from the Gamma-Ray Burst GRB 130427A, Science 343 (Jan., 2014) 38-41, [1311.5489]. 